\title{
Archivos de Prevención de Riesgos Laborales en MEDLINE/PubMed
}

\author{
Ana M. García, Jordi Delclós, Gero Domínguez, Laura Fontcuberta, \\ Miquel Mira, Fernando G. Benavides ${ }^{a}$
}

Con fecha de 2 de julio de 2012, en Archivos de Prevención de Riesgos Laborales recibíamos una carta firmada por Mr. Sheldon Kotzin, de la U.S. National Library of Medicine, en la que lo primero que buscamos rápidamente en el texto - de forma similar a como hacemos los autores con la carta que nos llega de la revista a la que hemos remitido un trabajo para su posible publicación- fue alguna frase que comenzara claramente con una expresión positiva. Y la encontramos, como a mitad del primer párrafo de la carta en cuestión:

"I am pleased to inform you that Archivos de Prevencion de Riesgos Laborales has been selected to be indexed and included in MEDLINE. Citations from the articles indexed, the indexing terms, and the English abstract printed in the journal will be included and searchable using PubMed"

Son muy buenas noticias para Archivos y para todas las personas involucradas en la revista, en todos los sentidos. Con este Editorial pretendemos dar a conocer a nuestra audiencia las implicaciones de este acontecimiento, aprovechando para ubicarlo en una larga trayectoria y un largo proceso que culmina (en realidad, también un proceso que comienza, confiemos que igualmente largo y productivo) con la carta de Mr. Kotzin.

La historia de Archivos de Prevención de Riesgos Laborales se remonta ya casi 50 años, íntimamente asociada a la biografía de la Societat Catalana de Seguretat i Medicina del Treball (SCSMT). En los antecedentes más remotos se encuentra la anterior publicación de la SCSMT, Medicina de Empresa, fundada en 1963 y que llegó a publicar 120 núme$\operatorname{ros}^{1}$. Su transformación en Archivos de Prevención de Riesgos Laborales se materializó en 1998, contando con el firme apoyo de la SCSMT y la vocación y entrega del primer director de la revista en esta nueva etapa ${ }^{2}$. Desde entonces, Archivos ha publicado con regularidad germana (si se nos permite todavía la expresión en estos tiempos que corren) un ejemplar cada tres meses, cumpliendo en 2012 quince años de existencia, en total 60 números. Actualmente todos los miembros de la SCSMT, junto a los de la Asociación de Medicina del Trabajo de la Comunidad Valenciana (AMTCV), reciben puntualmente la revista. En total se distribuyen alrededor de 2000 ejemplares a lectores e instituciones de España, Alemania, Estados Unidos de América, Canadá, Costa Rica, Gran Bretaña, Italia y Holanda.

Desde 2007, aunque este ejercicio ya contaba con antecedentes esporádicos en la revista, en el primer número de cada año de Archivos se presentan de forma regular los datos más significativos en relación con el proceso editorial. Según el último de estos informes ${ }^{3}$, Archivos publica trabajos procedentes de prácticamente todas las comunidades autónomas del Estado Español (destacando en número los autores de Cataluña y de la Comunidad Valenciana) y también de varios países de Latinoamérica. Las instituciones desde las que se elaboran los trabajos publicados incluyen administraciones laboral y sanitaria, servicios de prevención, mutuas y universidades, con predominio de estas últimas. Las páginas de la revista acogen trabajos que cubren el amplio espectro de temáticas propias de la salud laboral, incluyendo artículos de investigación sobre políticas preventivas, enfermedades laborales, evaluación de riesgos, vigilancia de la salud o evaluación de intervenciones, por citar algunas de las áreas más representadas.

A pesar de la satisfacción con la que nos permitimos presentar, año tras año, el trabajo y resultados del proceso editorial en la revista, somos conscientes de que Archivos es una publicación modesta, de las llamadas revistas "locales", entendiendo como tales revistas que recogen principalmente la producción científica de un ámbito territorial limitado, con escasa internacionalización, y con la mayoría de contenidos en una lengua distinta al inglés (aunque en el caso de Ar-

\author{
Correspondencia: \\ Ana M. García \\ Universitat de València \\ anagar@uv.es
}


chivos estamos hablando de un ámbito muy amplio, que potencialmente incluye todos los países y población de habla hispana). Sabemos que los resultados de Archivos en términos de volumen e impacto de los trabajos publicados son moderados; lo que en ningún momento ha sido impedimento para trabajar por mantener los indicadores de gestión editorial en los niveles mínimos de calidad exigibles a las revistas científicas más prestigiosas $2,4,5$. Pero las revistas pequeñas, como Archivos, y "locales", como Archivos, también son necesarias. La investigación en salud laboral en España es todavía una actividad débil, que creemos, sin embargo, fundamental para mejorar la práctica profesional, las estrategias y las políticas para la protección y mejora de la salud y seguridad en el trabajo ${ }^{6,7}$. Y con ese convencimiento, Archivos ha pretendido fomentar la investigación en nuestro medio, intentando estimular, formar y ayudar en lo que constituye el último e imprescindible paso para que la investigación sea útil en todo su potencial: la publicación. Desde estas páginas hemos intentado también, desde el principio, acercar a los lectores al mundo de la publicación $3,4,8-10$, no siempre bien conocido, con sus propias normas y exigencias.

Si la publicación con criterios de calidad es fundamental para completar el proceso de investigación, entendida como aportación de conocimiento original, relevante y riguroso -otro elemento imprescindible sería la transferencia de ese conocimiento a la práctica ${ }^{11}$, un proceso en el que entendemos la publicación científica es también necesaria, aunque no suficiente-, una adecuada divulgación de lo que se publica es imprescindible para llegar a las audiencias diana. Y al igual que son exigibles criterios de calidad en el proceso de gestión y decisión editorial de las revistas científicas, son también exigibles estos criterios en los medios de difusión de las publicaciones. Y hoy en día, el recurso más generalizado, riguroso y accesible para dar a conocer las publicaciones científicas en el campo de la salud es MEDLINE y, en particular, su versión online, PubMed.

MEDLINE es la principal base de datos de la U.S. National Library of Medicine (NLM). Según la última información disponible ${ }^{12}$ (la base se actualiza a diario), MEDLINE contiene alrededor de 19 millones de referencias a artículos publicados en revistas científicas del ámbito de las ciencias de la vida, con énfasis en las publicaciones biomédicas. En MEDLINE se incluyen y actualizan regularmente las citas de aproximadamente 5600 revistas científicas de todo el mundo, publicadas en 39 lenguas distintas. Pese a su dimensión internacional, sin embargo, MEDLINE concentra sus esfuerzos en el mundo anglosajón: casi la mitad (45\%) de los artículos citados proceden de revistas publicadas en los Estados Unidos de América, y casi la totalidad de los mismos (91\%) están publicados en inglés. Una búsqueda en el catálogo de la NLM identifica tan sólo 69 revistas publicadas en España (en este listado ya se incluye Archivos, el lector curioso puede comprobarlo entrando en http://www.ncbi.nlm.nih.gov/nlmcatalog) y actualmente indizadas en MEDLINE, apenas algo más del 1\% de todas las incluidas en la base. Existen también bases de datos bibliográficas nacionales y Archivos está desde hace años incluida en el Índice Médico Español, una base de revistas científicas también del ámbito de las ciencias de la salud que a nuestro parecer ha conocido mejores momentos, y en el Índice Bibliográfico Español en Ciencias de la Salud (IBECS), que mantiene mayor vitalidad. El IBECS es un producto de la Biblioteca Nacional de Ciencias de la Salud, vinculada al Instituto de Salud Carlos III, que según la información disponible permite búsquedas en algo más de 200 revistas españolas, en su mayoría activas ${ }^{13}$. Pero uno de los objetivos históricos de Archivos, desde el primer intento en el año 2003, ha sido su indización en MEDLINE. No ha sido un objetivo fácil.

Cualquier revista que se precie quiere hacer visibles sus contenidos en las bases de datos bibliográficas de mayor prestigio. Y cualquier base de datos bibliográfica que se precie es exigente a la hora de decidir si una revista merece o no ser seleccionada para su indización en la base. El IBECS, que es una base bibliográfica de ámbito local y comparativamente modesta, establece como requisitos indispensables para la inclusión de una revista la existencia de instrucciones a los autores (normas de publicación) de la revista, la publicación de un mínimo de doce artículos originales al año, la inclusión de resúmenes y palabras clave de los artículos publicados en español y en inglés y la existencia de un proceso de revisión externa en la gestión editorial de los trabajos publicados ${ }^{13}$. MEDLINE es mucho más exigente para la selección y la permanencia de las revistas en su base ${ }^{14,15}$. Para ello, MEDLINE cuenta con un comité especializado (el así llamado Literature Selection Technical Review Committee) que se reúne tres veces al año evaluando en cada ocasión alrededor de 150 revistas científicas de todo el mundo que solicitan su inclusión en esta base de datos. Sólo unas pocas lo consiguen (menos del 25\%). Y normalmente, el éxito requiere perseverancia, además de cumplir con los criterios de evaluación exigidos. Archivos ha llamado cuatro veces a las puertas de MEDLINE. En julio de 2011 remitimos la documentación del último intento. Y en julio de 2012 recibimos la respuesta favorable que ya conocen los lectores.

Como también se lee en esta respuesta, la forma más habitual de consultar los contenidos de MEDLINE es a través de PubMed. MEDLINE y PubMed son recursos muy parecidos, aunque no exactamente iguales ${ }^{16}$. MEDLINE es la base que nutre fundamentalmente PubMed, pero en PubMed también tenemos acceso a citas que todavía no se han incluido en MEDLINE, aunque están en proceso, y también algunas citas de revistas y libros que no se encuentran en MEDLINE, incluidas en PubMed bajo el criterio de la NLM. Una de las características de MEDLINE es que todas las citas que contiene tienen asignados términos del vocabulario $\mathrm{MeSH}$ (el mismo que pedimos a los autores de Archivos que utilicen para identificar las palabras clave en sus artículos), lo que facilita la recuperación de esos trabajos en las búsquedas bibliográficas. 
Según nos han informado, una vez Archivos ha sido aceptada para su indización, y en cuanto se haga llegar a la oficina técnica los datos correspondientes, en MEDLINE aparecerán los contenidos de Archivos desde el año en curso en adelante. Es decir, a partir del volumen 15 de la revista. Esta ventana inmensa al mundo exterior de lo que se publica en Archivos es un estímulo adicional para todos los que participamos en la construcción de cada número de la revista: editores, revisores y autores, también la secretaría técnica y la empresa publicadora (Gràfiques Alzamora). El siguiente reto para una revista científica y sus autores está sin duda todavía muy lejos, pero no lo descartamos: la inclusión de Archivos de Prevención de Riesgos Laborales en la base del Journal Citation Reports, uno de los productos estrella en el mundo de la publicación científica de la multinacional Thomson Reuters, fuente del también mundialmente famoso "factor de impacto" bibliográfico, un indicador al que permanecen muy atentas las principales revistas científicas de salud laboral, tanto como los mejores investigadores en el área ${ }^{17}$. Pero ésa es ya otra historia. Mientras tanto, disfrutemos del momento: tras más de una década trabajando por ello, tras tres intentos fallidos, en medio de una crisis económica generalizada a la que parece que nada es ajeno, tampoco el mundo de la publicación, Archivos de Prevención de Riesgos Laborales es la primera y única revista en español (y producida en España) incluida en la categoría "Occupational Medicine" de MEDLINE/PubMed (http://wwwcf.nlm.nih.gov/serials/journals/index.cfm), categoría que contiene un limitado listado de 32 revistas de todo el mundo, en su mayoría de Estados Unidos $(\mathrm{n}=11)$ y del Reino Unido ( $\mathrm{n}=7$ ).

Siempre lo decimos, con total convencimiento: Archivos debe su existencia misma y todo lo que pueda ser ahora y en el futuro, al trabajo y confianza de personas e instituciones: las sociedades profesionales que la apoyan (en este momento, la Societat Catalana de Seguretat i Medicina del Treball y la Asociación de Medicina del Trabajo de la Comunidad Valenciana), la secretaría técnica (siempre atenta e imprescindible), los revisores externos con su generosa dedicación de tiempo en la mejora de los artículos, los autores que nos envían los frutos de su trabajo, el equipo editorial. También solemos recordarlo: a nuestro entender, la principal razón de ser (sin duda, la más legítima) de las revistas científicas son los lectores, y confiamos en que en esta nueva etapa Archivos consiga llegar a muchos más profesionales e investigadores de la salud laboral en todo el mundo. Todos los que participamos y hacemos posible Archivos tenemos motivos para alegramos con este crecimiento en la vida de la revista, pero desde aquí queremos dedicar el logro muy especialmente a nuestros autores: la llegada de su trabajo a la base de datos bibliográfica en ciencias de la salud con mayor difusión y reconocimiento internacional es un justo reco- nocimiento a la confianza que depositan en la revista y al esfuerzo que supone, no sólo llevar a cabo y escribir un trabajo científico, sino también adaptarse a un proceso editorial que sabemos, también como autores, no suele ser especialmente complaciente.

Y por último, queremos aprovechar también desde estas páginas para invitar a participar en Archivos, una vez más ${ }^{1}$, a otras sociedades profesionales que quieran unir sus esfuerzos en el arduo trabajo por mantener una publicación científica con los estándares mínimos de calidad en un mundo pequeño, como el nuestro. Por nuestra parte, también como siempre, seguiremos en el empeño de publicar y difundir conocimiento válido, útil y riguroso.

\section{BIBLIOGRAFÍA}

1. De Montserrat i Nono J. Una nueva revista con una larga historia. Arch Prev Riesgos Labor. 1998:1:1-3.

2. Benavides FG. Una cultura científica para la prevención de riesgos laborales. Arch Prev Riesgos Labor. 1998:1:5-6.

3. Fontcuberta L, García AM, Delclòs J, Domínguez G. Archivos 2011: la regularidad como valor (y antídoto contra la crisis). Arch Prev Riesgos Labor. 2012;15:7-9.

4. García AM. Las normas de publicación en las revistas científicas. Arch Prev Riesgos Labor. 2004;7:131-3.

5. García AM. Por qué revistas científicas, por qué Archivos. Arch Prev Riesgos Labor. 2006;9:1-2.

6. Ronda E, Seguí MM, Bonfill X, Urrutia G, Serra C. Archivos Evidencia, una apuesta para la práctica de una salud laboral basada en la evidencia científica. Arch Prev Riesgos Labor. 2012;15:10-1.

7. Boix P, Rodríguez de Prada A. Buena práctica profesional y calidad de la prevención: una iniciativa de transferencia impulsada por el Instituto Nacional de Seguridad e Higiene en el Trabajo. Arch Prev Riesgos Labor. 2011;14:177-8.

8. Arranz Lázaro M. Como escribir y publicar un artículo científico. Arch Prev Riesgos Labor. 1998;1:33-6.

9. García AM, Delclòs J, Mira M. Archivos 2006. Arch Prev Riesgos Labor. 2007;10: 8-10.

10. García AM. Archivos Selección: una mirada hacia otras revistas. Arch Prev Riesgos Labor. 2007;10:171-2.

11. Boix P. Del conocimiento a la acción: importancia de la transferencia en salud laboral. Arch Prev Riesgos Labor. 2009;12:57-8.

12. U.S. National Library of Medicine. MEDLINE. Fact Sheet [actualizado 22 dic 2011] [citado 1 sep 2012]. Disponible en: http://www.nlm.nih.gov/pubs/factsheets/medline.html

13. Biblioteca Virtual en Salud. Búsqueda en bases de datos. IBECS [citado 2 sep 2012]. Disponible en: http://ibecs.isciii.es/

14. U.S. National Library of Medicine. MEDLINE Journal Selection. Fact Sheet [actualizado 3 ago 2012] [citado 1 sep 2012]. Disponible en: http://www.nlm.nih.gov/pubs/factsheets/jsel.html

15. Delgado López-Cózar E, Ruiz-Pérez R, Jiménez-Contreras E. Criterios MEDLINE para la selección de revistas científicas. Metodología e indicadores. Aplicación a las revistas médicas españolas con especial atención a las de Salud Pública. Rev Esp Salud Pública. 2006;80:52151.

16. U.S. National Library of Medicine. What's the Difference Between MEDLINE and PubMed? Fact Sheet [actualizado 9 abr 2010] [citado 2 sep 2012]. Disponible en: http://www.nlm.nih.gov/pubs/factsheets/dif_med_pub.html

17. Smith DR. Historical Development of the Journal Impact Factor and its Relevance for Occupational Health. Ind Health. 2007;45:730-42. 